\title{
Development of a Glass-Bead Device for Dissolution Testing
}

Marija Bogataj*, Greta Cof, and Aleš Mrhar

Faculty of Pharmacy, University of Ljubljana, Aškerčeva 7, Ljubljana, SI-1000 Ljubljana, Slovenia

e-mail: marija.bogataj@ffa.uni-lj.si

\begin{abstract}
A device for dissolution testing with a special type of stirring element and typical movement of a dosage form was developed and constructed in our laboratory. It consists of a working vessel in which a suitable amount of small glass beads is pushed by a stirring bar in an appropriate medium. A heap of beads forms in front of the stirring bar and moves around the vessel. A solid dosage form, most frequently a tablet, is placed in the medium on the surface of the bead layer and is moved due to the movement of this bead layer. The newly developed device could be used in experimental work as a part of a dissolution system based on the flow-through principle. Two different series of tablets containing different drugs were tested to show the applicability of this device. Dissolution from one series of tablets was strongly dependent on increasing mechanical influences on the tablet during dissolution testing, while dissolution from the tablets of another series was not susceptible to the change in the same mechanical parameters. Comparison with dissolution results in USP Apparatus 2 also showed differences between different stirring rates in the case of mechanically susceptible tablets, while tablets that were not susceptible to these influences gave very similar dissolution profiles in both systems. Mechanical influences on the dosage form in the newly developed system could be varied by changing the amount of glass beads and the stirring rate; however, the biorelevance of parameters of this system remains to be determined.
\end{abstract}

KEYWORDS: Dissolution; release; glass-bead dissolution device; solid dosage forms; nondisintegrating tablets.

\section{INTRODUCTION}

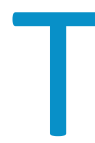

he development of novel dissolution approaches and devices, like the one described in the present article, represents a special challenge to the researchers working in the dissolution field. Namely, dissolution testing is one of the most frequently used biopharmaceutical tests for the evaluation of oral solid dosage forms. Absorption profiles can often be predicted on the basis of dissolution data if certain requirements, high permeability being the crucial one, are fulfilled. Another aspect that is very important for good prediction of in vivo performance relates to the conditions of dissolution testing, which should closely reflect the in vivo situation and can thus increase the probability of reliably predicting the absorption profiles.

Different apparatus and methods are used for dissolution testing, most frequently those described in pharmacopeias. Mechanical influences on the dosage form are different in these apparatus; most frequently, the dosage form is not in direct contact with the stirring element but is exposed only to the flow of liquid. In many cases, for example in USP Apparatus 1 and 2 (1, 2), mechanical influences on the dosage form can be varied using different rotation speeds of the stirring element. Some authors use modified pharmacopeial

*Corresponding author. apparatus where certain parts are adapted, for example, paddles with different shapes and dimensions. Thus, a new mode of stirring was introduced by Qureshi et al. (3) who used a curve-shaped spindle with bristles attached instead of a paddle in USP Apparatus 2. This approach was used with the purpose of improving stirring and preventing sedimentation in the dissolution vessel. Apart from pharmacopeial apparatus and their modifications, other systems are also available, such as the complex multicompartmental in vitro TIM system (4), which is able to simulate many conditions in the gastrointestinal (GI) tract. The mode of movement of the dosage form in the TIM system also differs significantly from conventional apparatus. In their dissolution system, Garbacz et al. (5) simulated the different pressure forces that influence the dosage form during its movement along the GI tract. There are other systems described in the literature (6) that simulate at least some of the physical influences to which the dosage form is exposed in the Gl tract up to a certain degree of biorelevance. Much information about different noncompendial dissolution methods that simulate different physiological parameters including physical forces is given in a recent review of McAllister (7). However, in spite of these different approaches, the fact remains that the mechanical influences to which the dosage form is exposed in the GI tract are very difficult to 
simulate with in vitro models.

In the in vivo system, that is, in GI tract after oral administration, the dosage form is exposed to different physiological media. A solid dosage form, for example the tablet, might float in these media but is probably frequently in direct contact with GI mucosa. The contact of the tablet with mucosa depends on many different parameters, among which volume of medium, which might frequently be relatively $\operatorname{low}(8,9,10)$, luminal caliber, wall tone, and contractions (11) are certainly very important. The anatomical features of the different parts of the GI tract to which the dosage form is exposed can be well visualized on endoscopic photographs (12). In general, we can say that during its passage through the Gl tract, the dosage form is exposed to mechanical influences due to the movement of the musculature of the Gl tract and to flows of the liquids present in its lumen.

The purpose of the present work was to develop a dissolution device where the dosage form is in physical contact with and moves with the surface of the solid material at the bottom of the vessel. To achieve our goal, a certain quantity of small glass beads was introduced into a medium in a working vessel and stirred with a magnetic stirrer. This device represents part of a simple flowthrough dissolution system in which some physiological conditions can be well simulated. In the present article, the basic principles of the device are described and its applicability is shown by testing different types of tablets. In future work, the physiological relevance of this concept will be determined.

\section{MATERIALS AND METHODS Materials}

Two series of tablets that differed in composition and size were chosen for evaluation in the dissolution device. The tablets also contained different model drugs: the first series of tablets contained diclofenac sodium (one tablet contained $100 \mathrm{mg}$ of diclofenac sodium) and the second series contained tramadol hydrochloride (one tablet contained $100 \mathrm{mg}$ of tramadol hydrochloride). On the basis of our previous work (13), these two series of tablets were expected to have different mechanical susceptibilities (i.e., tablets of the series containing diclofenac were expected to be highly mechanically susceptible, while tablets of the series containing tramadol were not).

USP simulated gastric fluid without enzymes, $\mathrm{pH} 1.2$, and simulated intestinal fluid without enzymes, $\mathrm{pH} 6.8$ (2) were used as dissolution media. All substances used for the preparation of dissolution media were of analytical grade.
The spherical glass beads had a diameter of $1 \mathrm{~mm}$ and a density of $2.5 \mathrm{~g} / \mathrm{cm}^{3}$. It was confirmed experimentally that the tested drugs dissolved in dissolution media did not bind to the surface of the glass beads.

\section{Methods}

\section{Dissolution Testing Using Glass-Bead Device}

The experiments were performed in a dissolution system constructed in our laboratory that works on an open flow-through principle $(13,14)$. The system was composed of a $150-\mathrm{mL}$ glass vessel in a thermostatcontrolled water bath on a magnetic stirrer (IKA-Werke, IKA, Staufen, Germany). Fresh medium was delivered into the vessel by the aid of a peristaltic pump (IKA-Werke, IKA, Staufen, Germany), and medium from the working vessel was pumped out by the same pump at the same flow rate. Thus, a constant flow of medium into and out of the vessel was maintained throughout the whole period of the experiment enabling the maintenance of a constant volume of medium in the working vessel. The conditions used in the experiments were as follows: the working vessel volume was $150 \mathrm{~mL}$, volume of the medium in the vessel was $40 \mathrm{~mL}$, the temperature was $37{ }^{\circ} \mathrm{C}$ and the flow rate was $2 \mathrm{~mL} / \mathrm{min}$. The stirring was performed by a magnetic stirrer at 50 rpm or higher (i.e., $100 \mathrm{rpm}$ and $150 \mathrm{rpm}$ in some experiments). The medium was pumped out of the vessel through a stainless steel screen $(250 \mu \mathrm{m})$ or through a cylindrical full-flow filter (70 $\mu \mathrm{m})$. A special mode of movement was achieved by this novel stirring device: a suitable quantity of spherical glass beads in the working vessel was stirred by a magnetic stirrer. The stirring bar had to be long enough to enable efficient stirring of the beads. A cylindrical stirring bar $50 \mathrm{~mm}$ long and $8 \mathrm{~mm}$ in diameter was used (the inner diameter of the vessel was $57 \mathrm{~mm}$ ) in the experiments. The quantity of beads ranged from 20 to $70 \mathrm{~g}$ in different experiments. At the beginning of each experiment, a tablet was put in the medium on the surface of the bead layer. The medium that flowed out of the working vessel was collected at 20-min intervals, and the amount of drug released was determined spectrophotometrically by measuring absorbance at $276 \mathrm{~nm}$ (diclofenac sodium) or $271 \mathrm{~nm}$ (tramadol hydrochloride). Dissolution tests were performed in medium consisting of simulated gastric fluid for $2 \mathrm{~h}$, and then the influx medium was changed to simulated intestinal fluid, which was used until the end of the experiment.

\section{Design of Glass-Bead Device Dissolution System with Four Working Stations}

A technically upgraded dissolution system with four working stations based on the glass-bead device was constructed by Merel d.o.o., Slovenia. Four working 
vessels are immersed in the water bath. A magnetic stirrer is mounted under each working vessel under the water bath, and the stirring rate is controlled separately for each vessel. The framework of the system enables easy mounting of the vessels and their centering on magnetic stirrers. The possible stirring rates that can be achieved are 1-150 rpm. Medium is pumped into and out of the working vessels by the aid of two peristaltic pumps (Masterflex L/S, Multichannel Cartridge Pump Head 7519-20), each of which maintains constant flow for two vessels. All testing conditions were completely the same as described above for the basic system with one working station.

\section{Dissolution Testing Using USP Apparatus 2}

Experiments were performed according to the requirements prescribed in USP. After the dissolution medium simulated gastric fluid was used for $2 \mathrm{~h}$, it was then drained from the vessel and replaced by simulated intestinal fluid, which was used until the end of the experiment. Stirring rates were 50 and $100 \mathrm{rpm}$; however, 150 and $200 \mathrm{rpm}$ were also used in some experiments to check the mechanical susceptibility of the tablets at a higher mechanical load.

\section{RESULTS AND DISCUSSION}

\section{Design of the Glass-Bead Dissolution Testing Device}

The glass-bead device, also called a peristaltic movementsimulating stirring device, is a dissolution testing device $(13,14)$ where a solid dosage form is in physical contact with the surface of the glass-bead layer and moves due to the movement of this layer. The aim of development of this device was to simulate some of the conditions to which a dosage form is exposed in the GI tract (i.e., physical contact with mucosa, movement of the dosage form due to the movement of mucosa, and possibly the exposure to the flows of liquid in the GI tract). How well the conditions simulate the in vivo situation remains an open issue and will be the topic of future research, while the scope of the present work is a description of the basic principles of this device and an evaluation of the parameters that might influence dosage form performance in this system.

The device is schematically represented in Figure 1, where three consecutive positions of the stirring element and the tablet can be seen. Three different, consecutive positions in the working vessel can also be seen from the photographs in Figure 2.

This very simple dissolution system was constructed to enable at least some of the critical conditions to mimic physiological ones. The main purpose of the novel dissolution device is to provide simultaneous gentle physical contact of a solid dosage form with the surface of the solid material at the bottom of the vessel and motion due to the movement of this material. This is achieved with a suitable amount of small inert beads, which settle at the bottom of the vessel and are stirred in a suitable medium. The beads are pushed by the stirring bar, thus forming an elevated mound in front of it. It appears that this mound of beads is moving around the vessel due to the movement of the stirring bar. The solid dosage form, most frequently a tablet, is placed in the medium; in most cases, it settles on the surface of the beads. Thus it is in contact with the surface of the glass-bead layer, it moves around the vessel due to the stirring action, and additionally it moves up and down due to the movement of the bead layer. This system is the most suitable for testing non-disintegrating tablets. Experiments can also be performed with pellets or with tablets that disintegrate; however, it is sensible only if particles of disintegrated tablets or pellets have appropriate size and density that prevent them from penetrating the glass-bead layer. Namely, inside the glass-bead layer, particles or pellets might be damaged due to strong mechanical forces that are certainly not physiological. The position of the tablet on the surface of the bead layer is also important. The tablet may be in contact with this layer only on one side or it may turn around, thus enabling physical contact with both sides. The latter is desirable; however, the behavior of the nondisintegrating tablet in the system depends on its properties. Some tablets start to float after a certain period of time and are in contact with the bead layer only occasionally. Floating could be the consequence of a change in tablet density due to swelling and dissolution of its components; however, for the same reasons, floating might also occur in vivo if a sufficient volume of liquid is available.

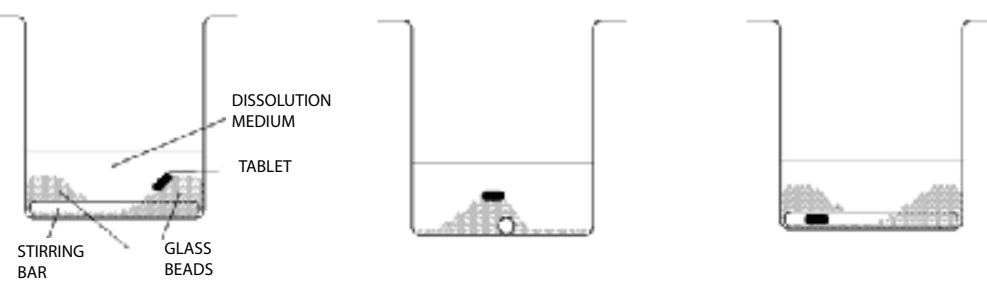

Figure 1. Schematic representation of the working vessel of the glass-bead device with the tablet at three consecutive positions. 

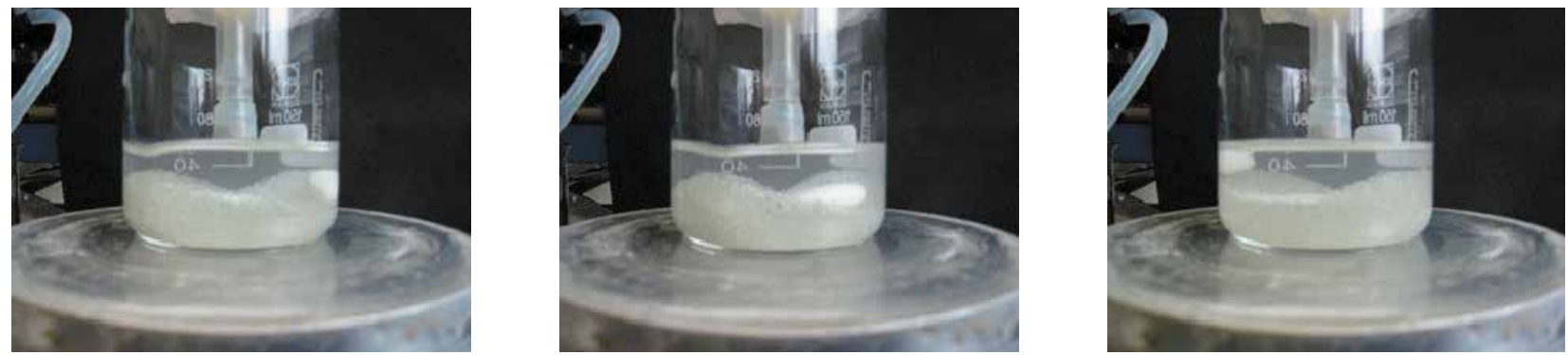

Figure 2. Working vessel of the glass-bead device during stirring with the tablet at three consecutive positions (water bath not shown).

\section{Control and Variability of Parameters of the Glass-Bead Device}

The first version of the dissolution device was constructed in our lab, and some settings were very difficult to control precisely. The following parameters were evaluated: pump flows were set and controlled at $2.0 \pm 0.01 \mathrm{~mL} /$ $\mathrm{min}$; the temperature in the working vessel was $37 \pm 0.5$ ${ }^{\circ} \mathrm{C}$ for the duration of the dissolution experiment; and the stirring rate on the magnetic stirrer was set and controlled by checking the values shown on the display; in the future, it will be controlled by suitable optical measuring device. The dissolution system with four working positions (Figure 3) was improved from a technical point of view. In this system working vessels were well fixed in the holes on the cover of the water bath, and exact centering of each vessel was enabled. Control of a much wider range of stirring rates, as in the first version of the system, was possible. Vessels, magnetic stirring bars, and glass beads used were the same as described above under description of the basic system.

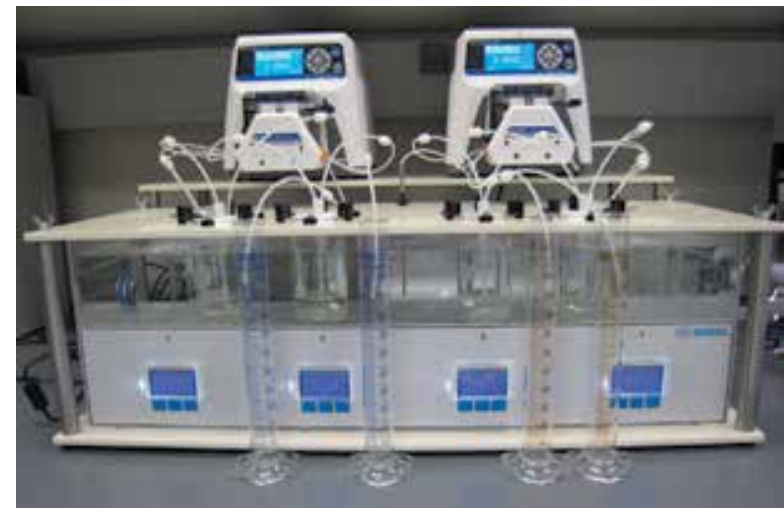

Figure 3. Open flow-through dissolution system with four working stations using the glass-bead device principle.

\section{Factors Influencing the Dissolution Process in the Glass-Bead Device}

The intensity and frequency of movement of the dosage form in the glass-bead system can be varied. The intensity of movement is strongly influenced by the quantity of beads used. On increasing the quantity of beads, the influence of the stirring bar and thus the intensity of movement of the bead layer surface are diminished and physical influences on the dosage form are lowered. The dissolution profiles of diclofenac sodium from the tablets using different amounts of glass beads are shown in Figure 4. As expected, a smaller amount of beads causes faster release of the drug due to the stronger mechanical influences to which the tablet is exposed.

The stirring rate can also be varied in the new system, and the dissolution rate increased with an increase in stirring rate. However, this had a smaller influence on drug dissolution from diclofenac tablets than the amount of beads. This is probably due to two opposite effects; namely, faster stirring intensifies the movement of the bead layer and also the dosage form. It is expected that this effect would produce faster dissolution, but at higher stirring rates many tablets float farther, thus decreasing physical contact with the bead layer and hence dissolution. The properties of the dosage form determine which of these two effects prevails. The influence of different stirring rates on the dissolution of diclofenac from tablets is shown in Figure 5.

Experiments with diclofenac tablets were also performed in USP Apparatus 2. On Figure 6A, the wide range of dissolution profiles obtained under different conditions using the glass-bead device can be seen in comparison with the dissolution profiles obtained in USP Apparatus 2 at $50 \mathrm{rpm}$ and $100 \mathrm{rpm}$. Slow and constant dissolution in simulated intestinal fluid in USP Apparatus 2 is evident, with a relatively small difference between the two stirring rates. Higher stirring rates (i.e., $150 \mathrm{rpm}$ and $200 \mathrm{rpm}$ ) are usually not used in dissolution testing in USP Apparatus 2 , as they can lead to a loss of discriminatory power. However, because the tablets showed a high mechanical susceptibility in the glass-bead device, we decided to increase the stirring rate in Apparatus 2 to 150 and 200 rpm just to evaluate tablet performance in a wide range 
of mechanical load. The results shown in Figure 6B confirm the high mechanical susceptibility of the tested tablets. However, this susceptibility can be seen much earlier using the more gentle conditions in the glass-bead device, while in the Apparatus 2 system, high mechanical susceptibility can be seen only after longer testing times or at unusually high stirring rates. Unfortunately, we have no data on the in vivo performance of the tested tablets and no evidence of their in vivo mechanical susceptibility. Thus, the biorelevance of the conditions in the glass-bead system remains an open issue that will have to be clarified in future studies.

For comparison, the same experiments were performed with tablets with a different composition containing tramadol hydrochloride. As stated above, these tablets were not expected to be mechanically susceptible, and this was confirmed by the dissolution profiles obtained at different conditions in the glass-bead device and in the Apparatus 2 system (Figure 7). No differences among the dissolution profiles obtained at different conditions (20$70 \mathrm{~g}$ glass beads and $50-150 \mathrm{rpm}$ ) in the newly developed system can be seen, and the differences in comparison with the profiles obtained in the Apparatus 2 system at $50 \mathrm{rpm}$ and $100 \mathrm{rpm}$ were also very small. Even when the stirring rate in the Apparatus 2 system was increased to 150 or $200 \mathrm{rpm}$ (not shown), only a small increase in dissolution rate was observed in comparison with lower stirring rates and with the profiles obtained in the glassbead device.

Thus, for a tablet that is susceptible to mechanical influences, very small differences in the mechanical load on the tablet in the glass-bead system resulted in different dissolution behavior, while for a tablet that is not mechanically susceptible, a wide variation in mechanical influences using different amounts of glass beads and stirring rates did not produce any changes in the dissolution profiles, which were also very close to those obtained in the Apparatus 2 system.

Thus, the dependence of drug dissolution from mechanically susceptible tablets on physical influences can be well analyzed in the novel glass-bead system. However, the quantitative values of both tested parameters (i.e., the amount of beads and the stirring rate that give the best simulation of in vivo conditions) remain to be evaluated on the basis of comparison with in vivo parameters. A comparison of the pellet dissolution data also obtained in the glass-bead system with the results of a pharmacokinetic study has already been performed (15). We expect that comparisons with other in vivo results and good in vitro-in vivo correlations between drug dissolution and absorption-plasma profiles after oral administration of different dosage forms will additionally confirm the suitability of this system for biorelevant dissolution testing.

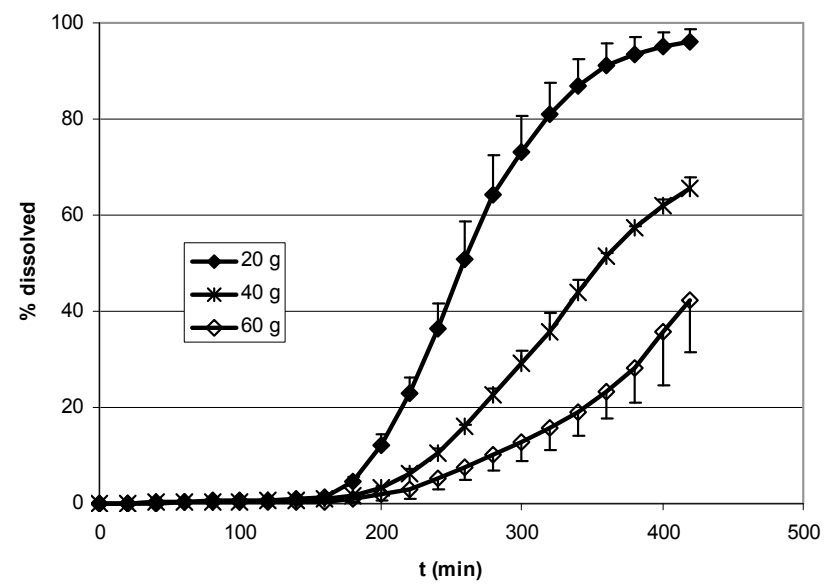

Figure 4. The influence of the amount of glass beads (legend) on the release of diclofenac sodium from tablets using glass-bead device.

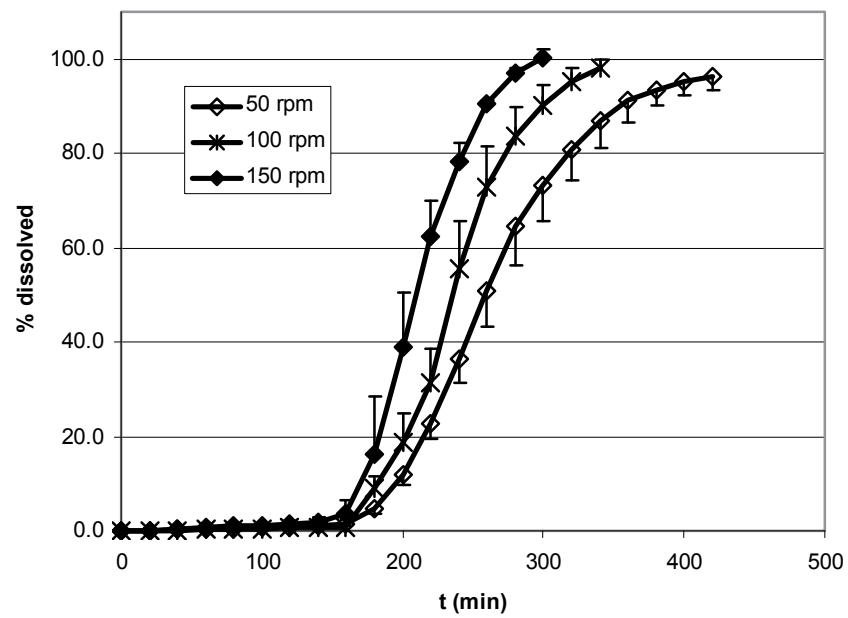

Figure 5. The influence of the stirring rate (legend) on the release of diclofenac sodium from tablets using glass-bead device. 

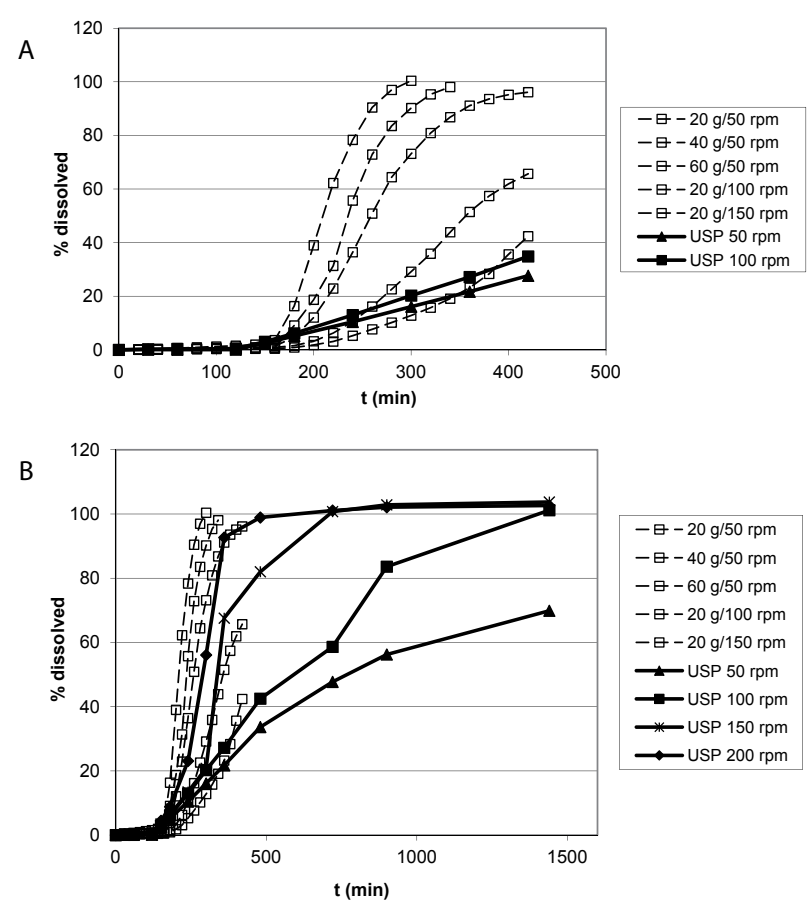

Figure 6. Comparison of dissolution profiles from tablets containing diclofenac sodium obtained using the glass-bead device at a wide range of different conditions (dashed lines, amount of glass beads and stirring rate shown in legend) with those obtained in the USP 2 system (full lines, stirring rate shown in legend). Figures $(A, B)$ differ in time scale and two additional USP profiles at $150 \mathrm{rpm}$ and $200 \mathrm{rpm}$ on Figure $6 B$.

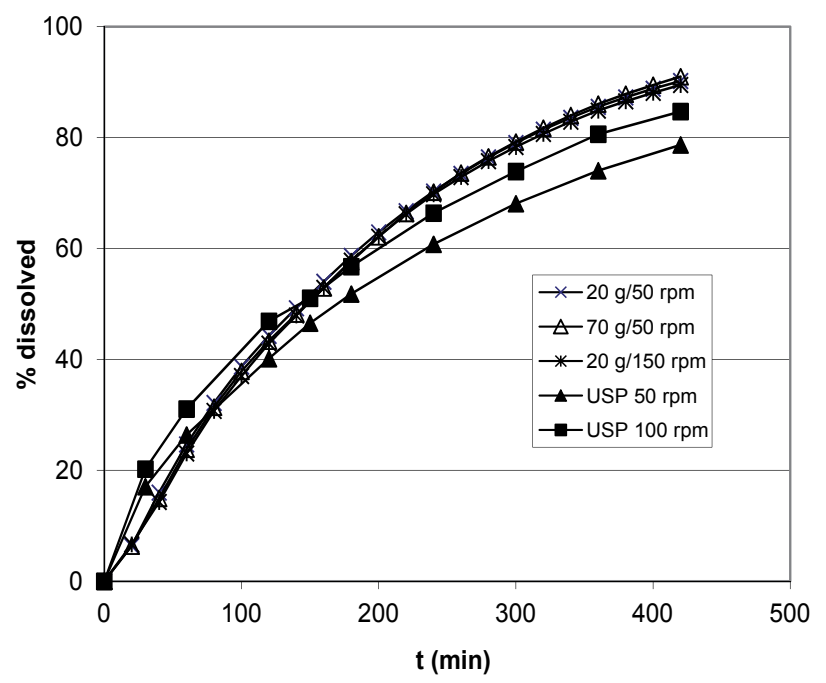

Figure 7. The influence of the amount of glass beads and stirring rate on the release of tramadol hydrochloride from tablets using the glass-bead device (empty symbols, amount of glass beads and stirring rate shown in legend) in comparison with USP 2 dissolution profiles obtained at $50 \mathrm{rpm}$ and $100 \mathrm{rpm}$ (full symbols, stirring rate shown in legend).

\section{CONCLUSION}

A device for dissolution testing using glass beads stirred by a magnetic stirrer in the dissolution medium was developed. In this system, a solid dosage form is in gentle physical contact with the surface of glass-bead layer and moves due to the movement of this layer. Physical influences on the dosage form, particularly on the tablet, may be studied by varying the amount of glass beads and the stirring rate; their influence on the dissolution profiles is dependent on the properties of the tablet, especially its mechanical susceptibility. However, the settings of parameters in the system have to be further evaluated and optimized, and the physiological relevance of the device needs to be confirmed by comparison with in vivo data.

\section{CONFLICT OF INTEREST}

No conflict of interest has been declared by the authors.

\section{REFERENCES}

1. European Pharmacopoeia, 7th ed.; European Directorate for the Quality of Medicines \& Healthcare, Council of Europe: Strasbourg, France, 2011.

2. The United States Pharmacopeia and National Formulary USP 35-NF 30; The United States Pharmacopeial Convention, Inc.: Rockville, MD, 2012.

3. Qureshi, S. A.; Shabnam, J. Applications of a new device (spindle) for improved characterization of drug release (dissolution) of pharmaceutical products. Eur. J. Pharm. Sci. 2003, 19 (4), 291-297. DOI: 10.1016/ S0928-0987(03)00120-9.

4. Blanquet, S.; Zeijdner, E.; Beyssac, E.; Meunier, J.-P.; Denis, S.; Havenaar, R.; Alric, M. A Dynamic Artificial Gastrointestinal System for Studying the Behavior of Orally Administered Drug Dosage Forms Under Various Physiological Conditions. Pharm. Res. 2004, 21 (4), 585-591. DOI: 10.1023/B:PHAM.0000022404 $.70478 .4 b$.

5. Garbacz, G.; Wedemeyer, R.-S.; Nagel, S.; Giessmann, T.; Mönnikes, H.; Wilson, C. G.; Siegmund, W.; Weitschies, W. Irregular absorption profiles observed from diclofenac extended release tablets can be predicted using a dissolution test apparatus that mimics in vivo physical stresses. Eur. J. Pharm. Biopharm. 2008, 70 (2), 421-428. DOI: 10.1016/J. EJPB.2008.05.029.

6. Mann, J. C.; Pygall, S. R. A Formulation Case Study Comparing the Dynamic Gastric Model with Conventional Dissolution Methods. Dissolution Technol. 2012, 19 (4), 14-19. DOI: 10.14227/ DT190412P14. 
7. McAllister, M. Dynamic Dissolution: A Step Closer to Predictive Dissolution Testing? Mol. Pharmaceutics 2010, 7 (5), 1374-1387. DOI: 10.1021/mp1001203.

8. Dubin, S. A.; Jense, H. G.; McCranie, J. M.; Zubar, V. Sugarless gum chewing before surgery does not increase gastric fluid volume or acidity. Can. J. Anesth. 1994, 41 (7), 603-606. DOI: 10.1007/BF03010000.

9. Lydon, A.; Murray, C.; McGinley, J.; Plant, R.; Duggan, F.; Shorten, G. Cisapride does not alter gastric volume or $\mathrm{pH}$ in patients undergoing ambulatory surgery. Can. J. Anesth. 1999, 46 (12), 1181-1184. DOI: 10.1007/BF03015530.

10. Schiller, C.; Fröhlich, C.-P.; Giessmann, T.; Siegmund, W.; Mönnikes, H.; Hosten, N.; Weitschies, W. Intestinal fluid volumes and transit of dosage forms as assessed by magnetic resonance imaging. Aliment. Pharmacol. Ther. 2005, 22 (10), 971-979. DOI: 10.1111/j.13652036.2005.02683.x.

11. Hasler, W. L. Small Intestinal Motility. In Physiology of the Gastrointestinal Tract, 4th ed.; Johnson, L. R., Ed.; Elsevier: Amsterdam, 2006; Vol. 1, pp 935-964.

12. Gastrolab Home Page. http://www.gastrolab.net/ (accessed July 15, 2015).

13. Bogataj, M.; Cof, G.; Mrhar, A. Mixing Device for Testing of Solution Level Imitating Peristaltic Movements (Mešalna naprava za testiranje raztapljanja, ki posnema peristaltično gibanje). Slovenian Patent SI 22853 A, February 26, 2010.

14. Bogataj M.; Cof, M.; Mrhar, A. Peristaltic movement simulating stirring device for dissolution testing. World Intellectual Property Organization patent application WO 2010/014046 A1, February 4, 2010.

15. Klein, S.; Garbacz, G.; Pišlar, M.; Locatelli, I.; Liu, C.; Weitschies, W.; Siegmund, W.; Mrhar, A.; Bogataj, $M$. The role of individual gastric emptying of pellets in the prediction of diclofenac in vivo dissolution. J. Controlled Release 2013, 166 (3), 286-293. DOI: 10.1016/j.jconrel.2012.12.032. 